\section{False-negative results on computer-aided detection software in preoperative automated breast ultrasonography of breast cancer patients}

\author{
Youngjune Kim ${ }^{1,2}$, Jiwon $\mathrm{Rim}^{1}$, Sun Mi Kim ${ }^{1,3}$, Bo La Yun ${ }^{1}$, So Yeon Park ${ }^{4}$, Hye Shin Ahn \\ Bohyoung $\mathrm{Kim}^{6}$, Mijung Jang ${ }^{1}$ \\ 1 Department of Radiology, Seoul National University Bundang Hospital, Seongnam; \\ ${ }^{2}$ Aerospace Medical Group, Air Force Education and Training Command, Jinju; ${ }^{3}$ Department \\ of Radiology, Seoul National University College of Medicine, Seoul; ${ }^{4}$ Department of \\ Pathology, Seoul National University Bundang Hospital, Seoul National University College \\ of Medicine, Seongnam; ${ }^{5}$ Department of Radiology, Chung-Ang University Hospital,Chung- \\ Ang University College of Medicine, Seoul; ${ }^{6}$ Division of Biomedical Engineering, Hankuk \\ University of Foreign Studies, Yongin, Korea
}

Purpose: The purpose of this study was to measure the cancer detection rate of computer-aided detection (CAD) software in preoperative automated breast ultrasonography (ABUS) of breast cancer patients and to determine the characteristics associated with false-negative outcomes.

Methods: A total of 129 index lesions (median size, $1.7 \mathrm{~cm}$; interquartile range, 1.2 to $2.4 \mathrm{~cm}$ ) from 129 consecutive patients (mean age \pm standard deviation, 53.4 \pm 11.8 years) who underwent preoperative ABUS from December 2017 to February 2018 were assessed. An index lesion was defined as a breast cancer confirmed by ultrasonography (US)-guided core needle biopsy. The detection rate of the index lesions, positive predictive value (PPV), and false-positive rate (FPR) of the CAD software were measured. Subgroup analysis was performed to identify clinical and US findings associated with false-negative outcomes.

Results: The detection rate of the CAD software was 0.84 (109 of 129; $95 \%$ confidence interval, 0.77 to 0.90 ). The PPV and FPR were 0.41 (221 of $544 ; 95 \% \mathrm{Cl}, 0.36$ to 0.45 ) and 0.45 (174 of $387 ; 95 \% \mathrm{Cl}, 0.40$ to 0.50 ), respectively. False-negative outcomes were more frequent in asymptomatic patients $(\mathrm{P}<0.001)$ and were associated with the following US findings: smaller size $(P=0.001)$, depth in the posterior third $(P=0.002)$, angular or indistinct margin $(P<0.001)$, and absence of architectural distortion $(\mathrm{P}<0.001)$.

Conclusion: The CAD software showed a promising detection rate of breast cancer. However, radiologists should judge whether CAD software-marked lesions are true- or false-positive lesions, considering its low PPV and high FPR. Moreover, it would be helpful for radiologists to consider the characteristics associated with false-negative outcomes when reading ABUS with CAD.

Keywords: Breast neoplasms; Computer-assisted detection; Ultrasonography;

Automated breast ultrasound

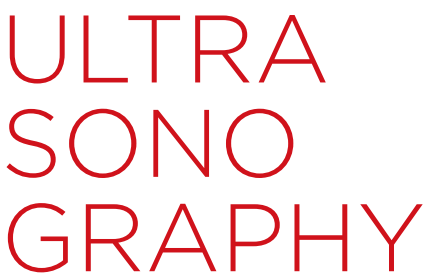

ORIGINAL ARTICLE

https://doi.org/10.14366/usg. 19076 pISSN: 2288-5919 • elSSN: 2288-5943 Ultrasonography 2021;40:83-92

Received: November 18, 2019

Revised: March 2, 2020

Accepted: March 24, 2020

Correspondence to: Mijung Jang, MD, PhD, Department of Radiology, Seoul National University Bundang Hospital, 82 Gumi-ro 173 beon-gil, Bundang-gu, Seongnam 13620, Korea

Tel. +82-31-787-7622

Fax. +82-31-787-4011

E-mail: mjjang74@gmail.com

This is an Open Access article distributed under the terms of the Creative Commons Attribution NonCommercial License (http://creativecommons.org/ licenses/by-nc/4.0/) which permits unrestricted noncommercial use, distribution, and reproduction in any medium, provided the original work is properly cited.

Copyright (C) 2021 Korean Society of Ultrasound in Medicine (KSUM)

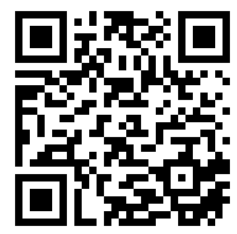

How to cite this article:

Kim Y, Rim J, Kim SM, Yun BL, Park SY, Ahn $\mathrm{HS}$, et al. False-negative results on computeraided detection software in preoperative automated breast ultrasonography of breast cancer patients. Ultrasonography. 2021 Jan;40(1):83-92. 


\section{Introduction}

Ultrasound (US) imaging of the breast, in addition to mammography and magnetic resonance imaging (MRI), plays a pivotal role in the screening and staging of breast cancer [1,2]. Handheld ultrasound (HHUS) is a standard imaging modality of the breast; however, HHUS has several drawbacks, such as a long examination time and low rate of reproducibility [3-5]. Automated breast ultrasound (ABUS) can overcome the shortcomings of HHUS [6-8] while maintaining diagnostic performance [9-12]. Furthermore, ABUS provides a wide field of view and coronal sections of the breast, which has the advantage of showing spiculated margins of breast cancer and architectural distortion $[10,13]$. ABUS is a promising modality for breast cancer screening in women with dense breast tissues [14,15], and several attempts have been made to use ABUS in the diagnostic setting [16-18].

Several studies have reported that computer-assisted detection (CAD) software may enhance the diagnostic performance of ABUS [19-22]. Improvement in the performance of CAD software has been observed with advances in machine learning algorithms [19]. However, to the best of our knowledge, no previous research has analyzed the shortcomings of ABUS CAD software in identifying breast cancer. Knowledge of the imaging features of breast cancers missed by the CAD software (false-negative cases) is crucial for optimizing the accuracy of diagnoses based on ABUS.

The purpose of this study was to measure the cancer detection rate of CAD software in preoperative ABUS of breast cancer patients and to determine the characteristics associated with false-negative outcomes.

\section{Materials and Methods}

Our institutional review board approved this study. The requirement for informed consent was waived because of the retrospective nature of the study.

\section{Patient Inclusion}

We included 147 consecutive breast cancer patients who underwent preoperative ABUS at our tertiary urban teaching hospital from December 2017 to February 2018. We did not analyze recurrent breast cancer in our study. We included both symptomatic and asymptomatic patients. At our institution, bilateral ABUS was routinely performed for preoperative evaluation of breast cancer patients, whereas HHUS was performed only in patients for whom ABUS was technically infeasible. Therefore, from the initial sample of 147 patients, those for whom ABUS was technically infeasible, those whose breast cancer was not visualized on ABUS, and those with bilateral breast cancers were excluded (Fig. 1). Among the 147 patients who were referred for preoperative ABUS during the study period, three patients in whom ABUS was technically infeasible due to the large mass of their breasts were excluded. Eleven additional patients whose breast cancers were not visualized on ABUS were excluded; in detail, these patients included one with occult breast cancer, two with status post-excisional biopsy, and eight patients with lesions not delineated on ABUS. Four other patients with bilateral breast cancers were excluded. Thus, we analyzed 129 index lesions (median size [interquartile range], $1.7 \mathrm{~cm}$ [1.2 to 2.4 $\mathrm{cm}$ ]; minimum, $0.4 \mathrm{~cm}$; maximum, $6 \mathrm{~cm}$ ) from 129 patients (mean age \pm standard deviation $[S D], 53.4 \pm 11.8$ years) with breast cancer visualized on ABUS.

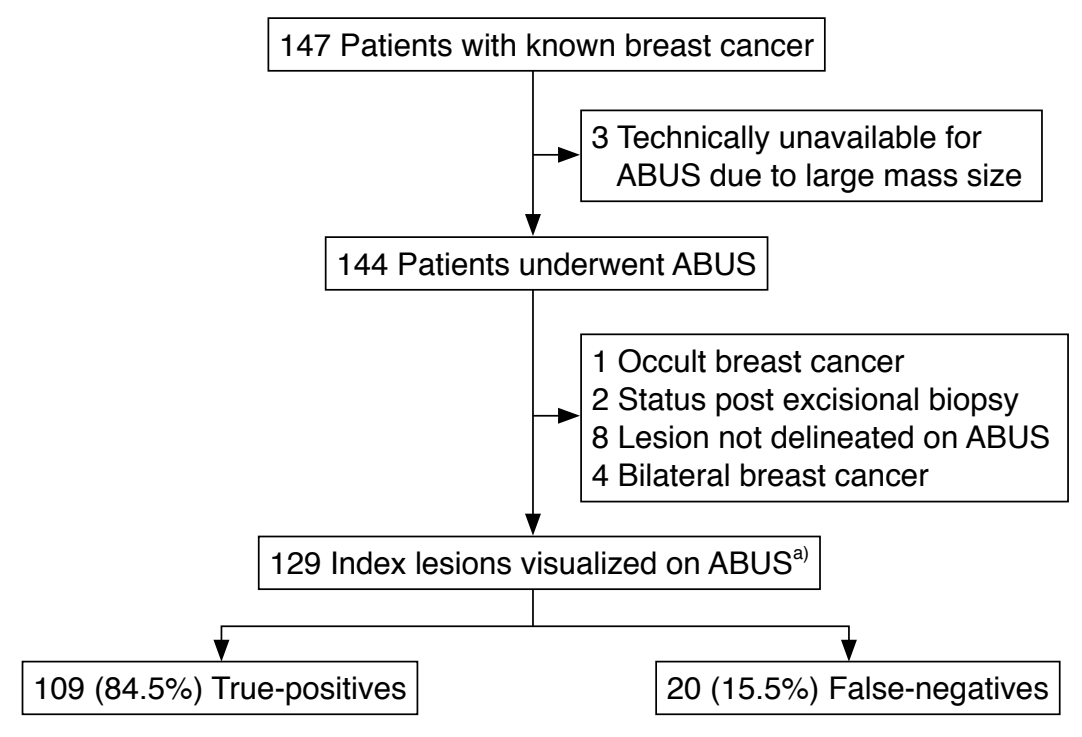

Fig. 1. Patient inclusion diagram. ABUS, automated breast ultrasonography. ${ }^{\text {a }}$ The index lesion was defined as a lesion confirmed as breast cancer by ultrasoundguided core needle biopsy. 


\section{ABUS Acquisition}

All ABUS examinations were performed using one of two $A B U S$ systems (Invenia ABUS, GE Healthcare, Sunnyvale, CA, USA) by one of two well-trained technologists. The examinations were performed with the patients in the supine position. A sponge was placed under the shoulder to help the breast tissue spread out evenly and the nipple face the ceiling. A nipple marker was placed for accurate coordination. An ABUS-specific lotion was applied to the breast to avoid contact artifacts. The level of breast compression was optimized for each patient by controlling the breast compression setting of ABUS, not only to spread out the breast evenly for better image quality, but also to maximize the patient's comfort.

The ABUS scan was continuous and automated with a 6-15$\mathrm{MHz}$ wide-aperture linear probe. Volumetric data were obtained in the axial plane with a slice thickness of $0.2 \mathrm{~mm}$ starting from the inferior portion of the breast. Coronal and sagittal images were reconstructed from the axial images. The field of view was set to $15.4 \mathrm{~cm} \times 17.0 \mathrm{~cm} \times$ up to $5 \mathrm{~cm}$ of depth from the skin to the chest wall. For each breast, three volumes were obtained: (1) the central volume with the nipple at the center of the footprint; (2) the lateral volume, which included the upper outer part of the breast tissue with the nipple located in the inferior-medial corner; and (3) the medial volume, which included the inner and inferior part of the breast tissue. Additional views were selectively obtained in patients with large breasts to avoid exclusion of tissue.

\section{ABUS CAD Software}

All volumetric data of ABUS were loaded onto a workstation dedicated for a deep learning-based commercialized CAD software (QVCAD version 2.1.2, QView Medical, Los Altos, CA, USA). The CAD software was applied to all ABUS examinations.

The output of the CAD software could be presented in two forms: (1) markers intended to highlight potentially malignant lesions and (2) minimum intensity projection images of the coronal section in areas where CAD software detected abnormalities. We only used $C A D$ markers to evaluate the diagnostic performance of the $C A D$ software (Fig. 2). The CAD markers did not display an exact value of the probability of malignancy potential.

The number of CAD markers displayed per ABUS volume could be adjusted by changing the values of the false-positive rate (FPR) in the configuration setting of the CAD software. According to the manual from the manufacturer [23], FPR was defined as the total number of false-positive CAD markers in non-cancer volumes divided by the total number of non-cancer volumes. In this study, we set the FPR to 0.2 (i.e., 1 false-positive CAD marker in non-cancer volume per 5 non-cancer volumes), which was its default setting.

\section{Image Interpretation}

Two breast radiologists (M.J. and S.M.K. with 12 and 16 years of experience in breast imaging, respectively, and 2 years of experience in ABUS) analyzed the 3D ABUS volume data at a dedicated ABUS workstation (Invenia ABUS Workstation, GE Healthcare). They thoroughly reviewed the ABUS images and recorded the most suspicious findings in the axial, coronal, and sagittal planes and reached consensus on their readings $[10,13]$.

First, the radiologists searched for an index lesion, which was defined as a mass confirmed as breast cancer by US-guided core needle biopsy. If a patient had multiple breast masses that were confirmed as breast cancer on the US-guided core needle biopsy, only the largest mass was regarded as the index lesion. We did not analyze non-mass lesions in this study. When searching for an index lesion on ABUS, the radiologists were not allowed to refer to the results of the CAD software. Instead, they were permitted to refer to clinical information and findings from available imaging such as HHUS performed during US-guided core needle biopsy, mammography, and preoperative MRI. After identifying the index lesion, the size, nipple-to-lesion distance, depth, shape, margin, and echo pattern of the lesion, as well as the background tissue echotexture and architectural distortion were recorded. If an index lesion was visualized in multiple ABUS volumes, the largest size of the index lesion and the shortest nipple-to-lesion distance were recorded. To determine the depth of the index lesion, the depth of the breast was divided into three parts: the anterior third, the middle third, and the posterior third of the fibroglandular tissue. In a lesion with more than 1 layer, the depth was determined by the layer on which the center of the lesion was located. The background tissue echotexture was measured using the method suggested by Kim et al. [24]. Otherwise, the findings on ABUS were assessed based on the Breast Imaging Reporting and Data System (BI-RADS) [25].

After the image interpretation, the radiologists checked whether the CAD marker correctly pointed to the index lesion on the ABUS volume data in the CAD software-dedicated workstation. For an index lesion visualized in multiple volumes of $A B U S$, they considered that the CAD marker indicated the index lesion correctly if the $C A D$ marker was placed at the index lesion on at least one ABUS volume.

\section{Electronic Medical Record Review}

Through a retrospective review of patients' electronic medical records, we recorded patients' age, menopausal status, family history of breast cancer, and symptoms. The final histopathology was determined by combining the pathological results of US-guided core needle biopsy and the surgical specimen. For the histopathological analysis, the index lesions were classified as invasive ductal carcinoma, invasive lobular carcinoma, and ductal carcinoma in 


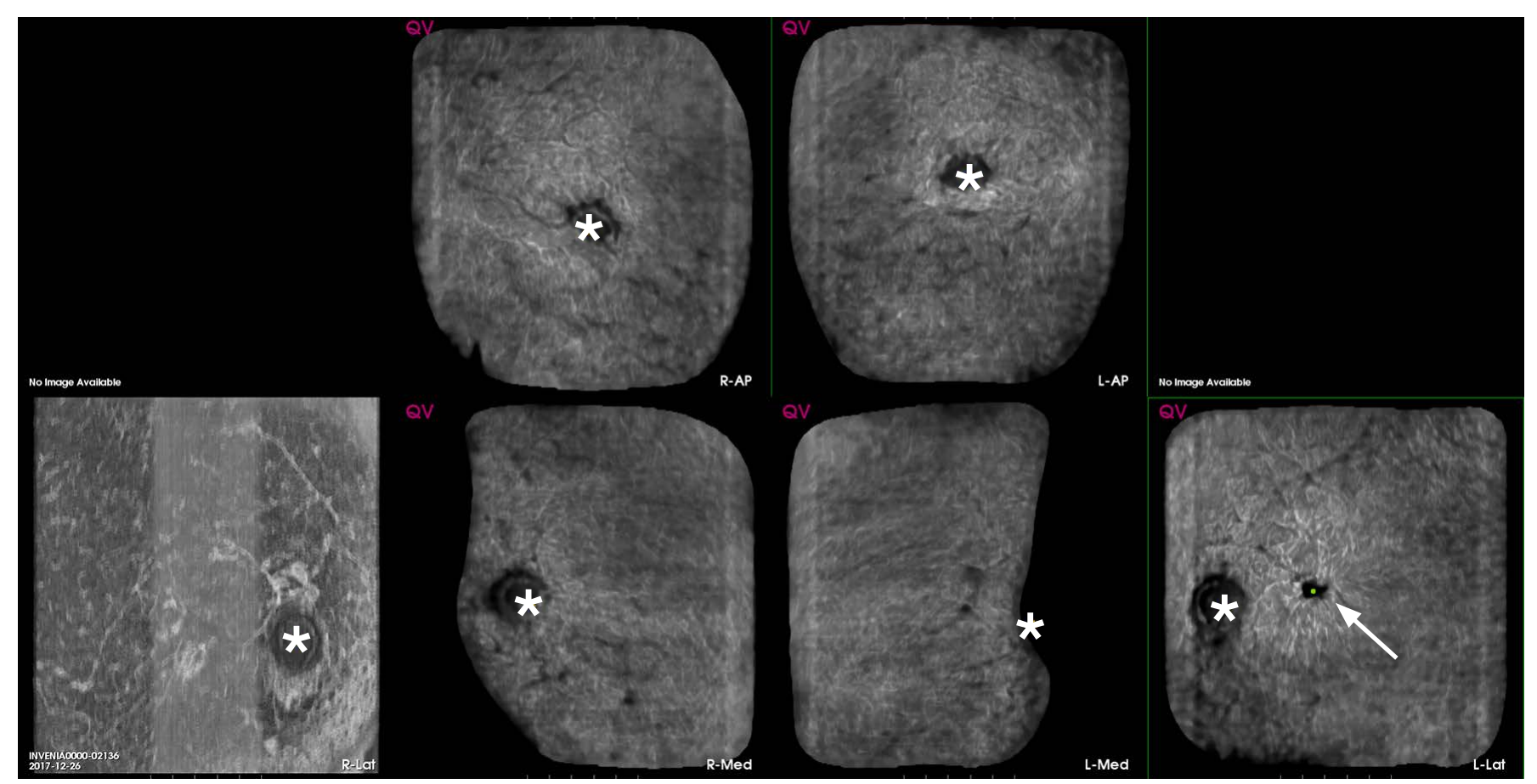

A

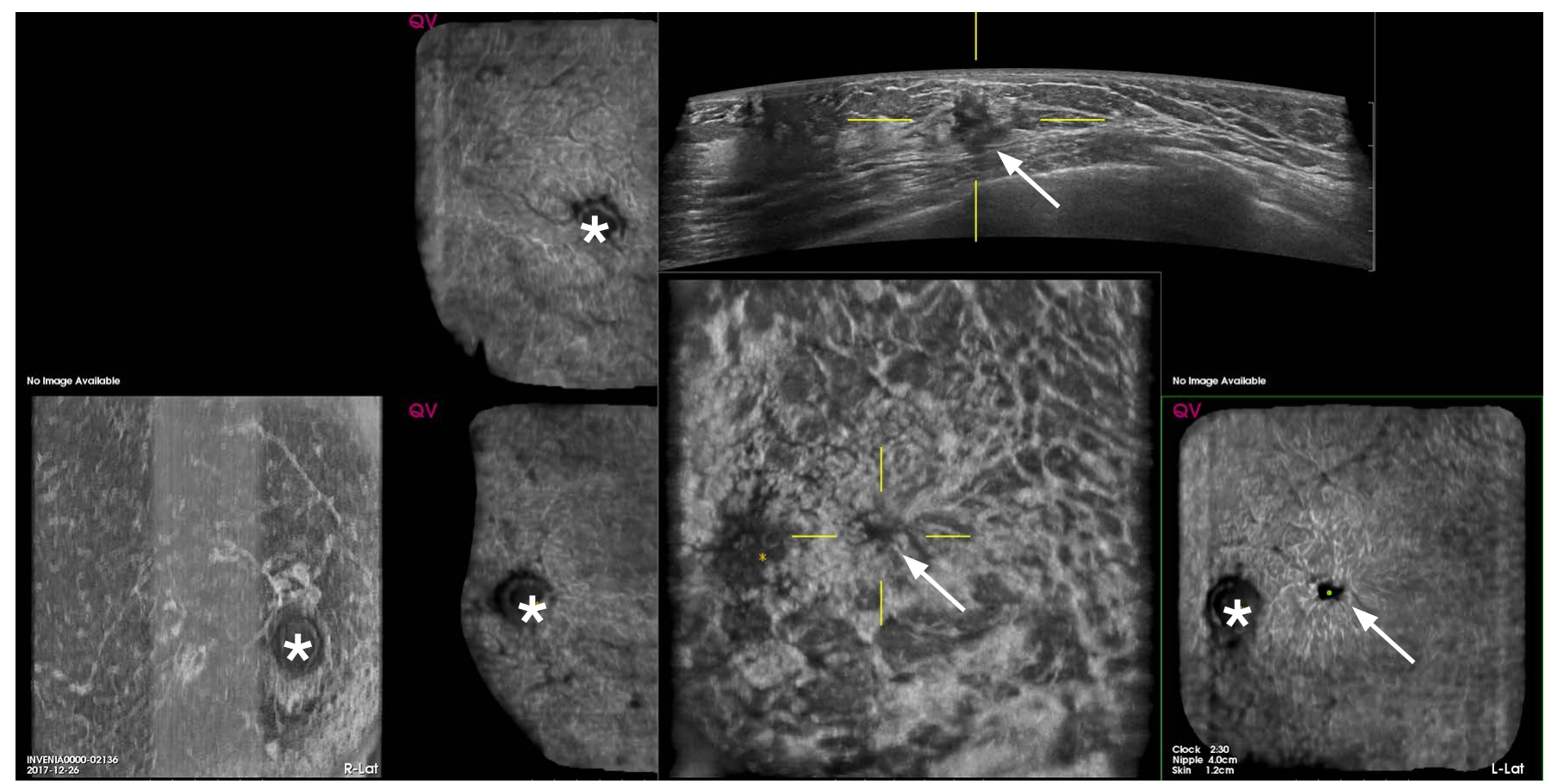

B

Fig. 2. A representative true-positive case (a 50-year-old patient with invasive ductal carcinoma detected by routine screening).

A. The computer-aided detection (CAD) software generated a marker (green dot) indicating potential malignant lesion (arrow). Asterisks denote nipple. B. The CAD software provided a quick review of automated breast ultrasonography images reconstructed in both the axial and coronal planes. The lesion was revealed to be invasive ductal carcinoma through ultrasound-guided biopsy. Asterisks denote nipple; arrows, potential malignant lesion; green dot, a marker generated by CAD software indicating potential malignant lesion. 
situ. A diagnosis of invasive cancer was made when an invasive component was found either in US-guided core needle biopsy or surgical specimens. Ductal carcinoma in situ was diagnosed if both US-guided core needle biopsy and surgical specimens were judged to be free from an invasive component. In cases wherein an immunohistochemistry examination was performed in both USguided core needle biopsy and surgical specimens, only the results from the US-guided core needle biopsy were used in the present study. The molecular subtype was classified into (1) hormone receptor (HR) positive and human epidermal growth factor receptor 2 (HER2) negative, (2) HER2 positive regardless of HR status, and (3) triple-negative/basal-like types.

\section{Data Analysis and Statistical Analysis}

The detection rate of index lesions of the CAD software on ABUS was measured. The detection rate was defined as the number of index lesions correctly indicated by the CAD marker divided by the total number of breast cancer patients in this study. Additionally, the positive predictive value (PPV) and FPR were measured to evaluate the diagnostic performance of the CAD software. PPV was defined as the number of markers properly located in the index lesion divided by the number of total markers in both non-cancer and cancer volumes. To check whether the FPR measured in our patient group was similar to the preset value of 0.2 in the CAD software, the definition of the FPR was identical to that in the manual of the CAD software [23] (i.e., the number of false-positive CAD markers in non-cancer volumes divided by the number of non-cancer volumes). In the present study, we measured the FPR using the ABUS volume data obtained from the contralateral side of breast cancer that was categorized as BI-RADS category 1 or 2 on preoperative ABUS. During the calculation of FPR, contralateral breasts that were classified as BI-RADS category 3 or above were excluded from the analysis. The results of preoperative MRI and US-guided core needle biopsy performed on the contralateral side of the breast were also checked to ensure the absence of breast cancer in the contralateral breast.

Subgroup analysis was performed to identify characteristics associated with false-negative outcomes of the CAD software. The Student $\mathrm{t}$ test and Fisher exact test were used for continuous and categorical variables, respectively. The tested variables were patients' age, menopausal status, family history of breast cancer, symptoms, histopathology, molecular subtype, and ABUS findings (size, nippleto-lesion distance, depth, shape, margin, and echo pattern of the lesion, background tissue echotexture, and architectural distortion).

All statistical analyses were performed using the open-source statistical software R (version 3.5.2, R Foundation for Statistical Computing, Vienna, Austria). Two-sided P-values of $<0.05$ were considered to indicate statistical significance.

\section{Results}

The detection rate of index lesions of the CAD software was 0.84 (109 of 129; 95\% confidence interval [CI], 0.77 to 0.90 ). The PPV and FPR of the CAD software were $0.41(221$ of $544 ; 95 \% \mathrm{Cl}, 0.36$ to 0.45 ) and 0.45 ( 174 of $387 ; 95 \% \mathrm{Cl}, 0.40$ to 0.50 ), respectively.

The baseline characteristics and histopathological results of the true-positive and false-negative groups are listed in Table 1. The presence of symptoms in the patients was significantly different between the two groups $(P<0.001)$; asymptomatic patients were more frequent in the false-negative group (true-positive vs. falsenegative, $20.2 \%$ [ 22 of 109 ] vs. $65.0 \%$ [ 13 of 20 ]). Otherwise, there were no significant differences in age, menopausal status, family history of breast cancer, histopathological findings, and molecular subtypes between the two groups.

The ABUS findings in the true-positive and false-negative groups are listed in Table 2. A smaller size of the index lesion was observed in the false-negative group (mean $\pm S D, 2.1 \pm 1.1 \mathrm{~cm}$ vs. $1.3 \pm 0.7$ $\mathrm{cm} ; \mathrm{P}=0.001)$. The depth of the lesion was significantly different between the two groups ( $P=0.002)$, as depth in the posterior third was more frequent in the false-negative group (5.5\% [6 of 109] vs. $25.0 \%$ [5 of 20]). The margin of the index lesion was associated with a lower cancer detection rate using the CAD software $(P<0.001)$, as angular margins $(6.4 \%$ [7 of 109$]$ vs. $35.0 \%$ [7 of 20]) and indistinct margins (28.4\% [31 of 109] vs. $45.0 \%$ [9 of 20]) were more frequent in the false-negative group. Absence of architectural distortion was associated with a higher falsenegative rate (24.8\% [27 of 109] vs. $90.0 \%$ [18 of 20], $P<0.001)$. A representative false-negative case is shown in Fig. 3.

\section{Discussion}

In this study, we measured the cancer detection rate of $C A D$ software in preoperative ABUS of breast cancer patients and analyzed the characteristics associated with false-negative outcomes. The detection rate of index lesions of the CAD software was 0.84 , and its PPV and FPR were 0.41 and 0.45 , respectively. False-negative outcomes were more frequent in asymptomatic patients and were associated with the following US findings: smaller lesion size on $A B \cup S$, depth in the posterior third, an angular or indistinct margin, and absence of architectural distortion.

The low PPV and high FPR observed in our study can be explained by the following reasons. First, a heterogeneous background tissue echotexture on ABUS was noted in a considerable portion of our included patients, which may lower the detection rate and increase 
Table 1. Baseline characteristics and histopathological results $(n=129)$

\begin{tabular}{|c|c|c|c|c|}
\hline Characteristic & Total $(n=129)$ & True-positive $(n=109)$ & False-negative $(n=20)$ & P-value \\
\hline Age (y) & $53.4 \pm 11.8$ & $53.5 \pm 11.9$ & $53.3 \pm 11.9$ & 0.956 \\
\hline Menopausal status & & & & 0.328 \\
\hline Premenopause & $53(41.1)$ & $47(43.1)$ & $6(30.0)$ & \\
\hline Postmenopause & $76(58.9)$ & $62(56.9)$ & $14(70.0)$ & \\
\hline Family history of breast cancer & & & & $>0.99$ \\
\hline Present & $17(13.2)$ & $15(13.8)$ & $2(10.0)$ & \\
\hline Absent & $112(86.8)$ & $94(86.2)$ & $18(90.0)$ & \\
\hline Symptom & & & & $<0.001^{*}$ \\
\hline No symptom & $35(27.1)$ & $22(20.2)$ & $13(65.0)$ & \\
\hline Lump & $91(70.5)$ & $84(77.1)$ & $7(35.0)$ & \\
\hline Nipple discharge & $1(0.8)$ & $1(0.9)$ & $0(0.0)$ & \\
\hline Nipple retraction & $2(1.6)$ & $2(1.8)$ & $0(0.0)$ & \\
\hline Histopathology & & & & 0.191 \\
\hline Invasive ductal carcinoma & $116(89.9)$ & 99 (90.8) & $17(85.0)$ & \\
\hline Ductal carcinoma in situ & $7(5.4)$ & $4(3.7)$ & $3(15.0)$ & \\
\hline Invasive lobular carcinoma & $4(3.1)$ & $4(3.7)$ & $0(0.0)$ & \\
\hline Inconclusive & $2(1.6)$ & $2(1.8)$ & $0(0.0)$ & \\
\hline Molecular subtype & & & & 0.066 \\
\hline $\mathrm{HR}+, \mathrm{HER} 2-$ & $91(70.5)$ & $77(70.6)$ & $14(70.0)$ & \\
\hline HER2+ & $10(7.8)$ & $6(5.5)$ & $4(20.0)$ & \\
\hline Triple-negative/basal-like & 24 (18.6) & $22(20.2)$ & $2(10.0)$ & \\
\hline Unknown & $4(3.1)$ & $4(3.7)$ & $0(0.0)$ & \\
\hline
\end{tabular}

Values are presented as mean \pm standard deviation or number (\%).

HR, hormone receptor; HER2, human epidermal growth factor receptor 2.

${ }^{*} \mathrm{P}<0.05$.

${ }^{a}$ Two patients were confirmed to have ductal carcinoma in situ on core needle biopsy but they were lost to follow-up before they underwent surgery. Thus, the absence of an invasive component in their breast cancers was not guaranteed due to the lack of a surgical specimen. For that reason, they were classified as having inconclusive

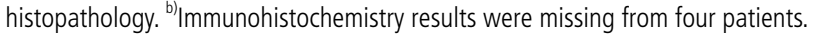

the FPR of CAD. Second, Asian women tend to have small and dense breasts. In a previous study performed in women with dense breasts, the PPV of the CAD software-based ABUS reading was 0.50 (95\% $\mathrm{Cl}, 0.45$ to 0.55 ) [26]. Moreover, there is a possibility of artifacts owing to insufficient compression. Third, while we set the FPR of our CAD based on data assuming a screening setting of a Western general population [23], our study results reflect the hypothetical screening setting of Asian breast cancer patients.

The smaller size of the index lesions led to a decrease in the cancer detection rate of the CAD software. Generally, it is known that small cancers can be overlooked on ABUS used in the screening setting [27]. A recent study that utilized the same CAD software as ours showed that the cancer detection rate of the ABUS CAD software was associated with tumor size, and the detection rate was around $90 \%$ for invasive ductal carcinoma larger than $1 \mathrm{~cm}$ [28]. Furthermore, Kim et al. [29] reported that a small size of the tumor $(<8 \mathrm{~mm})$ was associated with false-negative outcomes of the CAD software, although their CAD software was different from that used in the present study. This trend is maintained even when ABUS is interpreted by radiologists. A previous study reported that the detection rate of malignancy in the ABUS read by radiologists increased in proportion to the size of the lesion [30]. When the size of the target lesion was larger than $1.2 \mathrm{~cm}$, radiologists could reliably find the lesion [13]. Therefore, the results of the present study could reflect the weakness of ABUS itself in visualizing small masses.

Absence of architectural distortion was associated with a decreased cancer detection rate of the CAD software. Some articles demonstrated that 3D ABUS helped radiologists detect a spiculated margin of breast cancer or architectural distortion by showing the coronal section of the breasts $[10,13]$. Furthermore, it was suggested that the detection of architectural distortion may contribute to the 
Table 2. Findings of automated breast ultrasonography in patients with true-positives and false-negatives on computer-aided detection software $(n=129)$

\begin{tabular}{|c|c|c|c|}
\hline & True-positive $(n=109)$ & False-negative $(n=20)$ & P-value \\
\hline Size $(\mathrm{cm})$ & $2.1 \pm 1.1$ & $1.3 \pm 0.7$ & $0.001^{*}$ \\
\hline Nipple-to-lesion distance (cm) & $3.9 \pm 2.3$ & $4.5 \pm 2.6$ & 0.305 \\
\hline Anterior third & $25(22.9)$ & 0 & \\
\hline Middle third & $78(71.6)$ & $15(75.0)$ & \\
\hline \multicolumn{4}{|l|}{ BI-RADS ultrasonography lexicon } \\
\hline Shape & & & 0.781 \\
\hline Round or oval & $27(24.8)$ & $4(20.0)$ & \\
\hline Irregular & $82(75.2)$ & $16(80.0)$ & \\
\hline Margin & & & $<0.001^{*}$ \\
\hline Microlobulated & $39(35.8)$ & $1(5.0)$ & \\
\hline Spiculated & $29(26.6)$ & $2(10.0)$ & \\
\hline Echo pattern & & & 0.420 \\
\hline Hypoechoic & $72(66.1)$ & $12(60.0)$ & \\
\hline Isoechoic & $1(0.9)$ & $1(5.0)$ & \\
\hline Heterogeneous & $33(30.3)$ & $7(35.0)$ & \\
\hline Complex cystic and solid & $3(2.8)$ & $0(0.0)$ & \\
\hline Background tissue echotexture & & & 0.244 \\
\hline
\end{tabular}

Values are presented as mean \pm standard deviation or number (\%).

BI-RADS, Breast Imaging Reporting and Data System.

${ }^{*} \mathrm{P}<0.05$.

timely detection of breast cancer on ABUS [27]. Thus, the absence of architectural distortion might lower the diagnostic performance of the CAD software in detecting malignancy on ABUS.

Depth in the posterior third was associated with higher falsenegative rate of the CAD software. Lesions located in the deep posterior tissue are inherently difficult to visualize using transducers with a high insonating frequency due to attenuation of the US beam [31]. Moreover, detection of the masses in the deeper portion of the breast can be hindered by the nipple shadow or posterior shadowing of another lesion in a superficial location.

Angular and indistinct margins of the lesion were associated with a decreased cancer detection rate of the CAD software. Subtle noncircumscribed margins, artifacts, and architectural distortions may be difficult to capture on static images of US [31]. Moreover, an inability to freely adjust the degree of compression or scan angle could be a potential cause of false-negative outcomes on ABUS. Therefore, all available volume scan images must be sufficiently evaluated not only in the axial plane, but also in the coronal and sagittal planes, to overcome the false-negatives of the CAD software.

The CAD software used in the present study was based on a deep learning algorithm. Further improvement in the performance of the $C A D$ software might be achieved by modifying it to focus on the 


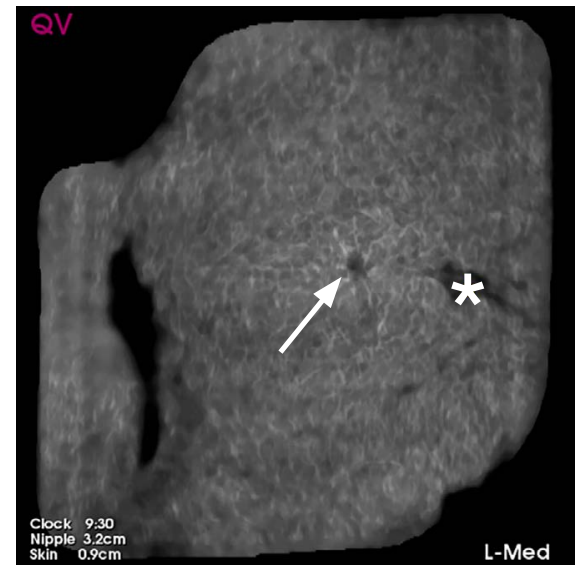

A

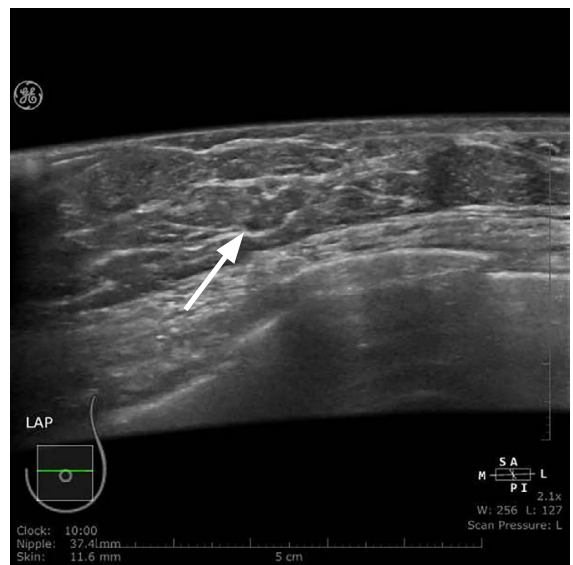

B

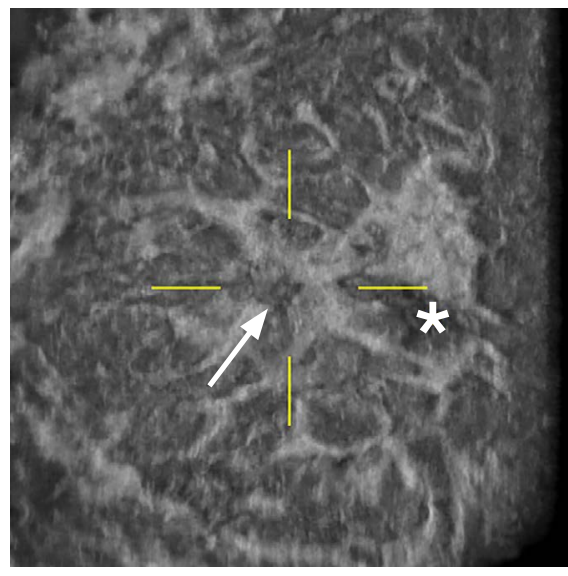

C

Fig. 3. A representative false-negative case (a 69-year-old woman with invasive ductal carcinoma detected by routine screening).

The lesion was confirmed as invasive ductal carcinoma through ultrasound-guided core needle biopsy. However, the CAD software did not point to the lesion appropriately. Subsequent left breast-conserving surgery was performed, and the final pathology report revealed a 1.3$\mathrm{cm}$ invasive ductal carcinoma (pT1c). A. The coronal view of automated breast ultrasonography (ABUS) shows a 1.2-cm irregular angular isoechoic mass (arrow) in the 9.5 o'clock direction, $3.2 \mathrm{~cm}$ from the nipple (asterisk) of the left breast. B. In the axial view of ABUS, the lesion was located in the posterior third of the left breast. B, C. In both axial and coronal views of ABUS, no definite architectural distortion was observed around the lesion (arrows). Asterisk represents the location of the nipple.

challenging factors revealed by our study (i.e., factors associated with false-negative outcomes). Promising results have been reported in recent studies of CAD applications using the latest deep learning algorithms [32,33].

The present study has several limitations. First, this was a singlecenter retrospective study with inherent limitations regarding its generalizability. Second, patients with breast cancer undetected on ABUS were excluded from the statistical analyses; however, this was due to the limitations of the ABUS device itself. Third, the radiologists who reviewed ABUS in our study were not blinded to the fact that they were assessing BI-RADS category 6 lesions. Fourth, all the ABUS scans were performed after core needle biopsy. Thus, the interpretation of ABUS might have been influenced by the findings of HHUS performed during the core needle biopsy. Furthermore, the biopsy itself may affect the margin and shape of lesion and may influence the detection rate of CAD software. Fifth, it is possible that interobserver variability in the lesion description may have influenced our results and may have decreased their reproducibility. Finally, we only included the largest mass in patients with multifocal or multicentric breast cancers in our analyses.

In conclusion, the CAD software showed a promising detection rate of breast cancer. However, radiologists should judge whether a CAD software-marked lesion is a true- or false-positive lesion, considering its PPV and high FPR. Moreover, it would be helpful for radiologists to keep in mind the characteristics associated with falsenegative outcomes when reading ABUS with CAD.
ORCID: Youngjune Kim: https://orcid.org/0000-0002-3698-6807; Jiwon Rim: https:// orcid.org/0000-0001-5278-6496; Sun Mi Kim: https://orcid.org/0000-0003-08993580; Bo La Yun: https://orcid.org/0000-0002-5457-7847; So Yeon Park: https:// orcid.org/0000-0002-0299-7268; Hye Shin Ahn: https://orcid.org/0000-0001-72607467; Bohyoung Kim: https://orcid.org/0000-0002-2183-5651; Mijung Jang: https:// orcid.org/0000-0001-9619-6877

\section{Author Contributions}

Conceptualization: Kim Y, Rim J, Kim SM, Park SY, Ahn HS, Kim B, Jang M. Data acquisition: Kim Y, Kim SM, Jang M. Data analysis or interpretation: Kim Y, Rim J, Kim SM, Yun BL, Park SY, Ahn HS, Kim $B$, Jang M. Drafting of the manuscript: Kim Y. Critical revision of the manuscript: Rim J, Kim SM, Yun BL, Park SY, Ahn HS, Kim B, Jang M. Approval of the final version of the manuscript: all authors.

\section{Conflict of Interest}

The CAD application (QVCAD; QView Medical, Los Altos, CA, USA) was used for research purposes only. We received no consulting fees from QView Medical, Inc.

\section{Acknowledgments}

This work was supported by Research Resettlement Fund for the new faculty of Seoul National University. The funders had no role in the study design, data collection and analysis, decision to publish, or preparation of the manuscript. 


\section{References}

1. Expert Panel on Breast Imaging, Mainiero MB, Moy L, Baron P, Didwania AD, diFlorio RM, et al. ACR Appropriateness Criteria ${ }^{\circledR}$ Breast Cancer Screening. J Am Coll Radiol 2017;14(11S):S383-S390.

2. Hortobagyi GN, Connolly JL, D'Orsi CJ, Edge SB, Mittendorf EA, Rugo HS, et al. Breast. In: Amin MB, Edge S, Greene F, Byrd DR, Brookland RK, Washington MK, et al., eds. AJCC cancer staging manual. 8th ed. New York, NY: Springer International Publishing, 2017;589-636.

3. Berg WA, Blume JD, Cormack JB, Mendelson EB, Lehrer D, Bohm-Velez $\mathrm{M}$, et al. Combined screening with ultrasound and mammography vs mammography alone in women at elevated risk of breast cancer. JAMA 2008;299:2151-2163.

4. Weigert J, Steenbergen $S$. The connecticut experiment: the role of ultrasound in the screening of women with dense breasts. Breast J 2012;18:517-522.

5. Berg WA, Blume JD, Cormack JB, Mendelson EB. Training the ACRIN 6666 Investigators and effects of feedback on breast ultrasound interpretive performance and agreement in BI-RADS ultrasound feature analysis. AJR Am J Roentgenol 2012;199:224-235.

6. Kelly KM, Dean J, Lee SJ, Comulada WS. Breast cancer detection: radiologists' performance using mammography with and without automated whole-breast ultrasound. Eur Radiol 2010;20:25572564.

7. Chang JM, Cha JH, Park JS, Kim SJ, Moon WK. Automated breast ultrasound system (ABUS): reproducibility of mass localization, size measurement, and characterization on serial examinations. Acta Radiol 2015;56:1163-1170.

8. Kaplan SS. Automated whole breast ultrasound. Radiol Clin North Am 2014;52:539-546.

9. Jeh SK, Kim SH, Choi JJ, Jung SS, Choe BJ, Park S, et al. Comparison of automated breast ultrasonography to handheld ultrasonography in detecting and diagnosing breast lesions. Acta Radiol 2016;57:162-169.

10. Vourtsis $A$, Kachulis A. The performance of $3 D$ ABUS versus HHUS in the visualisation and BI-RADS characterisation of breast lesions in a large cohort of 1,886 women. Eur Radiol 2018;28:592-601.

11. Chang JM, Moon WK, Cho N, Park JS, Kim SJ. Breast cancers initially detected by hand-held ultrasound: detection performance of radiologists using automated breast ultrasound data. Acta Radiol 2011;52:8-14.

12. Choi EJ, Choi H, Park EH, Song JS, Youk JH. Evaluation of an automated breast volume scanner according to the fifth edition of BI-RADS for breast ultrasound compared with hand-held ultrasound. Eur J Radiol 2018;99:138-145.

13. Shin HJ, Kim HH, Cha JH, Park JH, Lee KE, Kim JH. Automated ultrasound of the breast for diagnosis: interobserver agreement on lesion detection and characterization. AJR Am J Roentgenol 2011;197:747-754.

14. Brem RF, Tabar L, Duffy SW, Inciardi MF, Guingrich JA, Hashimoto $\mathrm{BE}$, et al. Assessing improvement in detection of breast cancer with three-dimensional automated breast US in women with dense breast tissue: the Somolnsight Study. Radiology 2015;274:663673.

15. Wilczek B, Wilczek HE, Rasouliyan L, Leifland K. Adding 3D automated breast ultrasound to mammography screening in women with heterogeneously and extremely dense breasts: report from a hospital-based, high-volume, single-center breast cancer screening program. Eur J Radiol 2016;85:1554-1563.

16. Chae EY, Shin HJ, Kim HJ, Yoo H, Baek S, Cha JH, et al. Diagnostic performance of automated breast ultrasound as a replacement for a hand-held second-look ultrasound for breast lesions detected initially on magnetic resonance imaging. Ultrasound Med Biol 2013;39:2246-2254.

17. Halshtok-Neiman $O$, Shalmon $A$, Rundstein $A$, Servadio $Y$, Gotleib M, Sklair-Levy M. Use of automated breast volumetric sonography as a second-look tool for findings in breast magnetic resonance imaging. Isr Med Assoc J 2015;17:410-413.

18. Shin HJ, Kim HH, Cha JH. Current status of automated breast ultrasonography. Ultrasonography 2015;34:165-172.

19. Tan T, Platel B, Mus R, Tabar L, Mann RM, Karssemeijer N. Computer-aided detection of cancer in automated 3-D breast ultrasound. IEEE Trans Med Imaging 2013;32:1698-1706.

20. Wang L, Bohler T, Zohrer F, Georgii J, Rauh C, Fasching PA, et al. A hybrid method towards automated nipple detection in 3D breast ultrasound images. Conf Proc IEEE Eng Med Biol Soc 2014;2014:2869-2872.

21. Schwaab J, Diez Y, Oliver A, Marti R, van Zelst J, Gubern-Merida A, et al. Automated quality assessment in three-dimensional breast ultrasound images. J Med Imaging (Bellingham) 2016;3:027002.

22. Park HJ, Kim SM, Yun BL, Jang M, Kim B, Jang JY, et al. A computeraided diagnosis system using artificial intelligence for the diagnosis and characterization of breast masses on ultrasound: added value for the inexperienced breast radiologist. Medicine (Baltimore) 2019;98:e14146.

23. QVCAD system user manual [Internet]. Los Altos, CA: QView Medical Inc., 2015 [cited 2020 Jan 15]. Available from: https:// www.accessdata.fda.gov/cdrh_docs/pdf15/P150043C.pdf.

24. Kim WH, Moon WK, Kim SJ, Yi A, Yun BL, Cho N, et al. Ultrasonographic assessment of breast density. Breast Cancer Res Treat 2013;138:851-859.

25. D'Orsi CJ, Sickles EA, Mendelson EB, Morris EA. ACR BI-RADS atlas: Breast Imaging Reporting and Data System. 5th ed. Reston, VA: American College of Radiology, 2013.

26. van Zelst JCM, Tan T, Clauser P, Domingo A, Dorrius MD, Drieling D, et al. Dedicated computer-aided detection software for automated 
3D breast ultrasound: an efficient tool for the radiologist in supplemental screening of women with dense breasts. Eur Radiol 2018;28:2996-3006.

27. van Zelst JC, Mann RM. Automated three-dimensional breast US for screening: technique, artifacts, and lesion characterization. Radiographics 2018;38:663-683.

28. Kim S, Kang BJ, Kim SH, Lee J, Park GE. Computer-aided detection with automated breast ultrasonography for suspicious lesions detected on breast MRI. Investig Magn Reson Imaging 2019;23:4654.

29. Kim JH, Cha JH, Kim N, Chang Y, Ko MS, Choi YW, et al. Computeraided detection system for masses in automated whole breast ultrasonography: development and evaluation of the effectiveness.
Ultrasonography 2014;33:105-115.

30. Chang JM, Moon WK, Cho N, Park JS, Kim SJ. Radiologists' performance in the detection of benign and malignant masses with 3D automated breast ultrasound (ABUS). Eur J Radiol 2011;78:99103.

31. Hooley RJ, Scoutt LM, Philpotts LE. Breast ultrasonography: state of the art. Radiology 2013;268:642-659.

32. Agarwal R, Diaz O, Llado X, Gubern-Merida A, Vilanova JC, Marti R. Lesion segmentation in automated 3D breast ultrasound: volumetric analysis. Ultrason Imaging 2018:40:97-112.

33. Yap MH, Pons G, Marti J, Ganau S, Sentis M, Zwiggelaar R, et al. Automated breast ultrasound lesions detection using convolutional neural networks. IEEE J Biomed Health Inform 2018;22:1218-1226. 